\title{
Azidopeptide Nucleic Acid. An Alternative Strategy for Solid Phase Peptide Nucleic Acid (PNA) Synthesis.
}

\author{
François Debaene, Nicolas Winssinger* \\ Institut de Science et Ingénierie Supramoleculaires, Université Louis Pasteur, 8 allée Gaspard Monge, \\ 67000 Strasbourg, France. \\ winssinger@isis.u-strasbg.fr
}

\section{Experimental Section}

General Techniques. All reactions were carried out under a nitrogen atmosphere with dry, freshly distilled solvents under anhydrous conditions, unless otherwise noted. Tetrahydrofuran (THF), toluene and diethyl ether $\left(\mathrm{Et}_{2} \mathrm{O}\right)$ were distilled from sodiumbenzophenone, and methylene chloride $\left(\mathrm{CH}_{2} \mathrm{Cl}_{2}\right)$ from calcium hydride. Anhydrous solvents were also obtained by passing them through commercially available alumina columns (Solv-Tek, Inc., VA). Yields refer to chromatographically and spectroscopically ( ${ }^{1} \mathrm{H}$ NMR) homogeneous materials, unless otherwise stated. Reagents were purchased at highest available commercial quality and used without further purification unless otherwise stated. Substituted polystyrene resins (100-200 mesh, 1\% DVB) were purchased from Advanced Chemtech. Reactions were monitored by thin layer chromatography (TLC) carried out on $0.25 \mathrm{~mm}$ E. Merck silica gel plates (60F-254) using UV light as visualizing agent and 10\% ethanolic phosphomolybdic acid or vanillin solution and heat, as developing agents. E. Merck silica gel (60, particle size 0.040-0.063 $\mathrm{mm}$ ) was used for flash column chromatography. NMR spectra were recorded on Brucker Advance-400 instruments and calibrated using residual undeuterated solvent as an internal reference. DMSO was calibrated to $2.5 \mathrm{ppm}$ for ${ }^{1} \mathrm{H} \mathrm{NMR}$ and $39.97 \mathrm{ppm}$ for ${ }^{13} \mathrm{C}$ NMR. The following abbreviations were used to explain the multiplicities: $s=$ singlet, $d$ $=$ doublet, $\mathrm{t}=$ triplet, $\mathrm{q}=$ quartet, $\mathrm{qt}=$ quintet, $\mathrm{m}=$ multiplet, $\mathrm{b}=$ broad. IR spectra were 
recorded on a Perkin-Elmer 1600 series FT-IR spectrometer. LC-MS were recorder using an Agilent 1100 HPLC with a Bruker micro-TOF instrument (ESI). Unless otherwise stated, a Supelco C8 ( $5 \mathrm{~cm} \times 4.6 \mathrm{~mm}, 5 \mu \mathrm{m}$ particules) column was used with a linear elution gradient from $100 \% \mathrm{H}_{2} \mathrm{O}\left(0.5 \% \mathrm{HCO}_{2} \mathrm{H}\right)$ to $100 \% \mathrm{MeCN}$ in 10 min at a flow rate of $0.5 \mathrm{~mL} / \mathrm{min}$

Benzyl 2-azidoethylglycinate (2). To a solution of 2-aminoethylbromide hydrobromide $(10.0 \mathrm{~g}, 48.8 \mathrm{mmol}, 1.0 \mathrm{eq})$ in DMF $(25 \mathrm{~mL})$ was added $\mathrm{NaN}_{3}(3.49 \mathrm{~g}, 53.7 \mathrm{mmol}, 1.1$ eq) and the reaction mixture was heated to $60^{\circ} \mathrm{C}$. After $3 \mathrm{~h}$, the reaction was cooled to 0 ${ }^{\circ} \mathrm{C}$ and triethylamine $(13.7 \mathrm{~mL}, 97.6 \mathrm{mmol}, 2.0 \mathrm{eq})$ was added followed by benzyl 2 bromoacetate $(6.21 \mathrm{~mL}, 39.0 \mathrm{mmol}, 0.8 \mathrm{eq})$. The reaction was stirred for an additional 2 $\mathrm{h}$ at $0{ }^{\circ} \mathrm{C}$ then diluted with $\mathrm{Et}_{2} \mathrm{O}(200 \mathrm{~mL})$ and washed with brine $(200 \mathrm{~mL})$. The organic phase was dried over $\mathrm{MgSO}_{4}$ and concentrated in vaccuo. The compound was then purified by flash chromatography (gradient, 30\% EtOAc in hexanes to $100 \%$ EtOAc) to obtain $5.87 \mathrm{~g}$ of 2 as a yellowish oil (64\% yield). $R_{f}=0.70$ (silica gel, EtOAc); FT-IR (neat) $v_{\max }, 3334.5,2940.4,2100.6,1739.8 \mathrm{~cm}^{-1} ;{ }^{1} \mathrm{H}$ NMR $\left(400 \mathrm{MHz}, \mathrm{D}_{6}-\mathrm{DMSO}, 25^{\circ} \mathrm{C}\right)$ $\delta$ 7.40-7.36 (m, 5H, Ar), $5.16\left(\mathrm{~s}, 2 \mathrm{H}, \mathrm{O}-\mathrm{CH}_{2}-\mathrm{C}\right), 3.46$ (s, $\left.2 \mathrm{H}, \mathrm{NH}-\mathrm{CH}_{2}-\mathrm{CO}\right), 3.34$ (t, $J=$ $\left.5.9,2 \mathrm{H}, \mathrm{N}_{3}-\mathrm{CH}_{2}-\mathrm{CH}_{2}\right), 2.78\left(\mathrm{t}, J=5.9,2 \mathrm{H}, \mathrm{N}_{3}-\mathrm{CH}_{2}-\mathrm{CH}_{2}\right) ;{ }^{13} \mathrm{C} \mathrm{NMR}(100 \mathrm{MHz}$, DMSO, $\left.25^{\circ} \mathrm{C}\right) \delta 172.5,136.5,128.8,128.5,128.4,65.9,50.9,50.3,48.1$; HRMS (ESI) calculated for $\mathrm{C}_{11} \mathrm{H}_{14} \mathrm{~N}_{4} \mathrm{O}_{2}:\left(\mathrm{MH}^{+}\right)=235.1111$, found: 235.1301 .

Thymine-1-acetic acid (3-T) was purchased from Aldrich (448958-25G)

4- $N$-(Benzhydryloxycarbonyl)cytosine-1-acetic acid (3-C). ${ }^{1}$ To cytosine (20.6 g, 180 mmol, 1.0 eq) in DMF $(180 \mathrm{~mL})$ was added potassium $t$-butoxide $(23.2 \mathrm{~g}, 207 \mathrm{mmol}$, $1.15 \mathrm{eq})$ and the reaction was heated to $100{ }^{\circ} \mathrm{C}$ for $2 \mathrm{~h}$. The reaction was then cooled to $10{ }^{\circ} \mathrm{C}$ and benzyl bromoacetate $(32.1 \mathrm{~mL}, 202 \mathrm{mmol}, 1.12 \mathrm{eq})$ was added drop-wise over $30 \mathrm{~min}$. The reaction was allowed to warm to room temperature while stirring for $12 \mathrm{~h}$, then quenched with acetic acid $(11.8 \mathrm{~mL}, 207 \mathrm{mmol}, 1.2 \mathrm{eq})$, concentrated and

\footnotetext{
${ }^{1}$ This compound was synthesized from cytosine according to a modified procedure from Coull et al. (patent WO 96/40685).
} 
thoroughly dried in vaccuo. The residues were re-suspended in $\mathrm{H}_{2} \mathrm{O}(200 \mathrm{~mL})$ and stirred for $4 \mathrm{~h}$ then filtered, washed with $\mathrm{H}_{2} \mathrm{O}(4 \times 300 \mathrm{~mL})$, dried under high vacuum to recover $41.2 \mathrm{~g}$ of benzyl cytosine-1-acetate as a pure compound ( $91.2 \%$ yield). To this material (41.2 g, $164 \mathrm{mmol}, 1.0 \mathrm{eq})$ dissolved in DMF $(320 \mathrm{~mL})$ was added carbonyldiimidazole (42.5 g, $262.5 \mathrm{mmol}, 1.6 \mathrm{eq}$ ). Progression of the reaction was monitored by TLC analysis of a reaction aliquot quenched with $\mathrm{MeOH}$. After $1.5 \mathrm{~h}$ of stirring, TLC analysis indicated that the isocyanate formation was complete and benzhydrol $(39.3 \mathrm{~g}, 213 \mathrm{mmol}$, 1.3 eq) was added. The reaction was heated at $60{ }^{\circ} \mathrm{C}$ and two more batches of benzhydrol ( 2 x $3.65 \mathrm{~g}, 19.8 \mathrm{mmol}, 0.12 \mathrm{eq})$ were added at $1 \mathrm{~h}$ intervals. After $6 \mathrm{~h}$, the heating was stopped and the reaction was allowed to proceed for $12 \mathrm{~h}$ and then quenched by the addition of $\mathrm{MeOH}(9.3 \mathrm{~mL}, 230 \mathrm{mmol}, 1.4 \mathrm{eq})$. The solvents were removed in vaccuo and the product was recrystallized from ethanol. The mother liquor was concentrated and recrystallized from $180 \mathrm{~mL}$ of $3 / 1 \mathrm{MeOH} / \mathrm{H}_{2} \mathrm{O}$. The two batches of impure powder were combined and further crystallized in methanol $(230 \mathrm{~mL})$ to recover $58.7 \mathrm{~g}(125 \mathrm{mmol})$ of pure benzyl (4- $N$-(benzhydryloxycarbonyl)cytosine)-1-acetate (76\% yield). This material $(58.7 \mathrm{~g}, 125 \mathrm{mmol}, 1.0 \mathrm{eq})$ was dissolved in acetonitrile:MeOH: $\mathrm{H}_{2} \mathrm{O}: \mathrm{EtOH}(2: 2: 1: 1,755 \mathrm{~mL})$ with heat and then cooled to $0{ }^{\circ} \mathrm{C}$ and treated with $\mathrm{LiOH}_{2} \mathrm{O}(51 \mathrm{~g}, 1.22 \mathrm{~mol}, 9.7 \mathrm{eq})$ dissolved in water $(393 \mathrm{~mL})$. The progression of the reaction was carefully monitored by TLC and quenched immediately upon consumption of the starting material with citric acid (117 g, $607 \mathrm{mmol}, 4.9 \mathrm{eq})$ in water $(580 \mathrm{~mL})$ to recover $44.3 \mathrm{~g}$ (117 mmol) of (4- $\mathrm{N}$-(benzhydryloxycarbonyl)cytosine)1-acetic acid 3-C (93\% yield). $R_{f}=0.15\left(30 \% \mathrm{MeOH}\right.$ in EtOAc); FT-IR (neat) $v_{\max }$ 3422.0, 3151.8, 3063.4, 1764.2, 1708.6, $1673.0 \mathrm{~cm}^{-1}$; ${ }^{1} \mathrm{H}$ NMR (400 MHz, D6-DMSO, $\left.25^{\circ} \mathrm{C}\right) \delta 8.03-8.01\left(\mathrm{~d}, J=7.5,1 \mathrm{H}, C_{6}\right), 7.46(\mathrm{~d}, J=7.5,4 \mathrm{H}, \mathrm{Ph}), 7.38(\mathrm{t}, J=7.5,4 \mathrm{H}$, $\mathrm{Ph}), 7.3(\mathrm{~d}, J=7.5,2 \mathrm{H}, \mathrm{Ph}), 6.96\left(\mathrm{~d}, J=7.5,1 \mathrm{H}, C_{5}\right), 6.82\left(\mathrm{~s}, 1 \mathrm{H}, \mathrm{CH}-\left(\mathrm{C}_{6} \mathrm{H}_{5}\right)_{2}\right), 4.50$ (s, $\left.2 \mathrm{H}, \mathrm{N}-\mathrm{CH}_{2}-\mathrm{CO}\right) ;{ }^{13} \mathrm{C} \mathrm{NMR}\left(100 \mathrm{MHz}, \mathrm{D}_{6}\right.$-DMSO, $\left.25^{\circ} \mathrm{C}\right) \delta 169.9,163.5,155.5,152.8$, 151.1, 140.8, 129.0, 128.3, 126.9, 94.2, 71.9, 51.3; HRMS (ESI) calculated for $\mathrm{C}_{20} \mathrm{H}_{17} \mathrm{~N}_{3} \mathrm{O}_{5}:\left(\mathrm{MN}_{\mathrm{a}}{ }^{+}\right): 402.1060$, found: 402.1010 .

6- $N$-(Benzhydryloxycarbonyl)adenine-9-acetic acid (3-A) ${ }^{1}$ Adenine $(20.0 \mathrm{~g}, 148$ mmol, $1.0 \mathrm{eq})$ in DMF $(350 \mathrm{~mL})$ was treated with sodium hydride $(60 \%$ in mineral oil, 
$6.88 \mathrm{~g}, 172 \mathrm{mmol}, 1.2 \mathrm{eq}$ ) in two portions at $1 \mathrm{~h}$ intervals. After $3 \mathrm{~h}$, all hydrogen gas evolution had stopped and the solution was cooled in an ice bath and benzylbromoacetate ( $26.6 \mathrm{~g}, 163 \mathrm{mmol}, 1.1 \mathrm{eq}$ ) was added drop-wise over $30 \mathrm{~min}$ The reaction was allowed to warm to room temperature while stirring for $12 \mathrm{~h}$ after which solvents were removed in vaccuo and water $(160 \mathrm{~mL})$ was added. After brief stirring, the water was decanted and the remaining sticky oil was dissolved in boiling ethanol and stirred overnight to obtain $29.3 \mathrm{~g}$ (99 mmol) of benzyl adenine-9-acetate as a solid (72\% yield). This crude material was dissolved in DMF $(200 \mathrm{~mL})$, treated with carbonyldiimidazole (24.0 g, $148 \mathrm{mmol}, 1.5 \mathrm{eq}$ ), heated slowly until $105^{\circ} \mathrm{C}$ and stirred at that temperature for $2 \mathrm{~h}$. The temperature was then decreased to $95^{\circ} \mathrm{C}$ before adding benzhydrol $(27.3 \mathrm{~g}, 148$ $\mathrm{mmol}, 1.5 \mathrm{eq}$ ) and the reaction was stirred for $12 \mathrm{~h}$ without further heating. The reaction was quenched with the addition of water $(500 \mathrm{~mL})$ and stirred vigorously for $2 \mathrm{~h}$ after which the solution was decanted and the residues dried under high vacuum. The crude oil was then redissolved in ethanol/acetonitrile $(1 / 1,330 \mathrm{ml})$ with heating and once a homogenious solution was obtained, water $(146 \mathrm{~mL})$ was added and the solution was cooled to $10{ }^{\circ} \mathrm{C}$. $\mathrm{LiOH}_{2} \mathrm{O}(74.9 \mathrm{~g}, 1.79 \mathrm{~mol}, 17.7 \mathrm{eq})$ in water $(365 \mathrm{~mL})$ was then added. The progression of the reaction was carefully monitored by TLC and quenched immediately upon consumption of the starting material with a solution of citric acid (192 g, $0.99 \mathrm{~mol}, 10 \mathrm{eq})$ in water $(400 \mathrm{~mL})$, stirred $10 \mathrm{~min}$ after which more water $(100 \mathrm{~mL})$ was added, and the mixture was cooled at $4{ }^{\circ} \mathrm{C}$ and allowed to crystallized overnight to afford $30.5 \mathrm{~g}$ of 6-N-(benzhydryloxycarbonyl)adenine-9-acetic acid (3-C) as a pure white solid $\left(76.4 \%\right.$ yield for 2 steps). $R_{f}=0.13\left(30 \% \mathrm{MeOH}\right.$ in EtOAc); FT-IR (neat) $v_{\max }$ 3297.9, 3058.3, 1736.0, $1614.7 \mathrm{~cm}^{-1}$; ${ }^{1} \mathrm{H}$ NMR $\left(400 \mathrm{MHz}, \mathrm{D}_{6}\right.$-DMSO, $\left.25{ }^{\circ} \mathrm{C}\right) \delta 10.9(\mathrm{~s}$, 1H, C-NH-CO), $8.63\left(\mathrm{~s}, 1 \mathrm{H}, C_{2}\right), 8.45\left(\mathrm{~s}, 1 \mathrm{H}, C_{8}\right), 7.54(\mathrm{~d}, J=7.5,4 \mathrm{H}, \mathrm{Ph}), 7.39$ (t, $J=$ $7.5,4 \mathrm{H}, \mathrm{Ph}), 7.3(\mathrm{t}, J=7.5,2 \mathrm{H}, \mathrm{Ph}), 6.83$ (s, $\left.1 \mathrm{H}, \mathrm{O}-\mathrm{CH}-\left(\mathrm{C}_{6} \mathrm{H}_{5}\right)_{2}\right), 5.09$ (s, $2 \mathrm{H}, \mathrm{N}-\mathrm{CH}_{2^{-}}$ $\left.\mathrm{CO}_{2} \mathrm{H}\right) ;{ }^{13} \mathrm{C}$ NMR $\left(100 \mathrm{MHz}\right.$, D6-DMSO, $\left.25{ }^{\circ} \mathrm{C}\right) \delta 169.5,152.6,152.1,151.6,149.8$, 145.3, 141.3, 128.9, 128.1, 126.9, 123.1, 77.7, 44.7; HRMS (ESI) calculated for $\mathrm{C}_{21} \mathrm{H}_{17} \mathrm{~N}_{5} \mathrm{O}_{4}:\left(\mathrm{MH}^{+}\right): 404.1275$, found: 404.1231 .

2- $N$-(Benzhydryloxycarbonyl)guanine-9-acetic $\quad$ acid $\quad$ (3-G). ${ }^{1} \quad$ To 2 -amino-6chloropurine $(27.9 \mathrm{~g}, 165 \mathrm{mmol}, 1.0 \mathrm{eq})$ in DMF $(280 \mathrm{~mL})$ at $85^{\circ} \mathrm{C}$ was added potassium 
carbonate (3.34 g, $247 \mathrm{mmol}, 1.5 \mathrm{eq})$. After brief stirring, the reaction mixture was cooled in an ice bath and benzyl-2-bromo-acetate $(28.0 \mathrm{~mL}, 177 \mathrm{mmol}, 1.07 \mathrm{eq})$ was added drop-wise, in three batches over $3 \mathrm{~h}$ while maintaining the temperature at $0{ }^{\circ} \mathrm{C}$. The reaction was then allowed to warm to room temperature and stirred for $12 \mathrm{~h}$ after which, the reaction mixture was filtered to remove the salts and the product precipitated by pouring the reaction mixture in vigorously stirring acidic water $(150 \mathrm{~mL}$ of $1 \mathrm{~N} \mathrm{HCl})$. After $2 \mathrm{~h}$ of stirring, a pink powder was filter and washed with water $(1.5 \mathrm{~L})$, then crystallized overnight from boiling acetonitrile while stirring $(280 \mathrm{~mL})$. The product was collected by filtration and washed with methanol and diethyl ether to yield $35.3 \mathrm{~g}$ (111 mmol) of benzyl 2-amino-6-chloropurine-9-acetate were obtained as white powder (67.7\% yield). This product was dissolved in THF $(435 \mathrm{~mL})$ cooled to $0{ }^{\circ} \mathrm{C}$ and triphosgene (11.9 g, $40.1 \mathrm{mmol}, 0.36 \mathrm{eq})$ was added. The reaction was stirred $0{ }^{\circ} \mathrm{C}$ for $1 \mathrm{~h}$ before adding drop-wise diisopropylethylamine (42.7 mL, $245 \mathrm{mmol}, 2.2 \mathrm{eq})$. After another $30 \mathrm{~min}$ at $0{ }^{\circ} \mathrm{C}$, benzhydrol (24.6 g, $\left.133.8 \mathrm{mmol}, 1.2 \mathrm{eq}\right)$ was added and the reaction was stirred for $12 \mathrm{~h}$ while warming to room temperature. The reaction was quenched with ethanol $(75 \mathrm{~mL})$ and concentrated until dryness in vaccuo. The oil was dissolved in $\mathrm{CH}_{2} \mathrm{Cl}_{2}$ and stirred with $10 \%$ aq citric acid $(200 \mathrm{~mL})$, after which the organic phase was washed with $5 \%$ sodium bicarbonate $(100 \mathrm{~mL})$, dried with $\mathrm{MgSO}_{4}$ and concentrated. The residues were recrystallized in boiling methanol $(300 \mathrm{~mL})$ to recovered $37.5 \mathrm{~g}$ (71.1 mmol) of benzyl 2- $N$-(benzhydryloxycarbonyl)-6-chloropurine-9acetate $(63.8 \%$ yield) as a pure solid. THF $(425 \mathrm{~mL})$ loaded with sodium hydride $(60 \%$ in mineral oil, $13.8 \mathrm{~g}, 345 \mathrm{mmol}, 5.0 \mathrm{eq})$ was cooled to $-78{ }^{\circ} \mathrm{C}$ before adding 3hydroxypropionitrile $(23.6 \mathrm{~mL}, 345 \mathrm{mmol}, 5.0 \mathrm{eq})$. After $2.5 \mathrm{~h}$ of stirring at $0{ }^{\circ} \mathrm{C}, 2-\mathrm{N}-$ (benzhydryloxycarbonyl)-6-chloropurine-9- $N$-acetate $(36.4 \mathrm{~g}, 68.96 \mathrm{mmol}, 1.0 \mathrm{eq})$ was added and the reaction mixture was further stirred for $12 \mathrm{~h}$ at room temperature. The THF was removed in vaccuo and the reaction mixture thus obtained was poured in $\mathrm{H}_{2} \mathrm{O}$ (250 mL), which was acidified until pH 3 with $20 \%$ aq citric acid to obtain a white precipitate. The precipitate was collected by filtration, washed with water and recrystallized 3 times in methanol to recover $25.1 \mathrm{~g}$ of 2-N(benzhydryloxycarbonyl)guanine-acetic acid 3-G (86.5\% yield). $R_{f}=0.18(30 \% \mathrm{MeOH}$ in EtOAc); FT-IR (neat) $v_{\max } 3422.0,3229.8,2925.5,1686.1,1611.1 \mathrm{~cm}^{-1}$; ${ }^{1} \mathrm{H}$ NMR 
(400 MHz, D6-DMSO, $\left.25^{\circ} \mathrm{C}\right) \delta 7.94\left(\mathrm{~s}, 1 \mathrm{H}, C_{8}\right), 7.48(\mathrm{~d}, 4 \mathrm{H}, \mathrm{Ph}, J=7.5), 7.39$ (t, 4H, $\mathrm{Ph}, J=7.5), 7.31$ (t, $2 \mathrm{H}, \mathrm{Ph}, J=7.5), 6.88\left(\mathrm{~s}, 1 \mathrm{H}, \mathrm{O}-\mathrm{CH}-\left(\mathrm{C}_{6} \mathrm{H}_{5}\right)_{2}\right), 4.87$ (s, $2 \mathrm{H}, \mathrm{N}-\mathrm{CH}_{2^{-}}$ $\left.\mathrm{CO}_{2} \mathrm{H}\right) ;{ }^{13} \mathrm{C}$ NMR $\left(100 \mathrm{MHz}\right.$, D6-DMSO, $\left.25{ }^{\circ} \mathrm{C}\right) \delta 169.9,169.5,155.5,154.1,149.7$, 147.6, 140.7, 140.5, 138,4, 129.3, 128.4, 126.9, 78.5, 44.9; HRMS (ESI) calculated for $\mathrm{C}_{21} \mathrm{H}_{17} \mathrm{~N}_{5} \mathrm{O}_{5}:\left(\mathrm{MH}^{+}\right): 420.1224$, found: 420.1281 .

Benzyl $N$-(2-azidoethyl)- $N$-(thymine-1-acetyl)glycinate 4-T. To a solution of thymine1-acetic acid 3-T (5.97 g, $32.4 \mathrm{mmol}, 1.0 \mathrm{eq})$ in DMF (35.0 mL) was added benzyl 2azidoethylglycinate $2(7.59 \mathrm{~g}, 32.4 \mathrm{mmol}, 1.0 \mathrm{eq})$, 4-DMAP (0.39 g, $3.24 \mathrm{mmol}, 0.1 \mathrm{eq})$, and EDCI ( $7.46 \mathrm{~g}, 38.9 \mathrm{mmol}, 1.2 \mathrm{eq}$ ). After $2 \mathrm{~h}$, the reaction was diluted with $\mathrm{CH}_{2} \mathrm{Cl}_{2}$ $(50 \mathrm{~mL})$ and washed with $\mathrm{HCl} 1 \mathrm{M}(50 \mathrm{~mL})$ and saturated $\mathrm{NaHCO}_{3}(100 \mathrm{~mL})$. Each aqueous phase was back extracted with $\mathrm{CH}_{2} \mathrm{Cl}_{2}(2 \times 100 \mathrm{~mL})$. The combined organic phases were dried over $\mathrm{MgSO}_{4}$ and concentrated to recover $12.2 \mathrm{~g}$ of spectroscopically pure 4-T as a white powder (94\% yield). $R_{f}=0.75(10 \% \mathrm{MeOH}$ in EtOAc); FT-IR (neat) $v_{\max } 3162.5,3032.8,2128.8, \quad 1746.5,1458.0 \mathrm{~cm}^{-1}$; ${ }^{1} \mathrm{H}$ NMR $(400 \mathrm{MHz}$, D6DMSO, $\left.25^{\circ} \mathrm{C}\right) \delta 7.37(\mathrm{~m}, 5 \mathrm{H}, \mathrm{Ar}), 7.24\left(\mathrm{~s}, 1 \mathrm{H}, \mathrm{C}_{6}\right), 5.23$ (s, 0.7H, O-CH${ }_{2} \mathrm{Ar}$, rotamer 1), $5.15\left(\mathrm{~s}, 1.3 \mathrm{H}, \mathrm{O}-\mathrm{CH}_{2}-\mathrm{Ar}\right.$, rotamer 2), $4.74\left(\mathrm{~s}, 1.3 \mathrm{H}, \mathrm{N}-\mathrm{CH}_{2}-\mathrm{CO}\right.$, rotamer 1$), 4.54(\mathrm{~s}$, $0.7 \mathrm{H}, \mathrm{N}-\mathrm{CH}_{2}-\mathrm{CO}$, rotamer 2), $4.48\left(\mathrm{~s}, 0.7 \mathrm{H}, \mathrm{N}-\mathrm{CH}_{2}-\mathrm{CO}\right), 4.18$ (s, $1.3 \mathrm{H}, \mathrm{N}-\mathrm{CH}_{2}-\mathrm{CO}$, rotamer2), $3.64\left(\mathrm{~m}, J=4.8,1.3 \mathrm{H}, \mathrm{N}_{3}-\mathrm{CH}_{2}-\mathrm{CH}_{2}\right.$, rotamer 1$), 3.50\left(\mathrm{t}, J=5.4,0.3 \mathrm{H}, \mathrm{N}_{3^{-}}\right.$ $\mathrm{CH}_{2}-\mathrm{CH}_{2}$, rotamer 2), 3.61 (t, $J=4.8,1.3 \mathrm{H}, \mathrm{N}_{3}-\mathrm{CH}_{2}-\mathrm{CH}_{2}-\mathrm{N}$, rotamer 1), 3.44 (t, $J=5.4$, $0.3 \mathrm{H}, \mathrm{CH}_{2}-\mathrm{CH}_{2} \mathrm{-N}$, rotamer 2) $\underline{8}: 1.77$ (s, $\left.3 \mathrm{H}, \mathrm{C}-\mathrm{CH}_{3}\right) ;{ }^{13} \mathrm{C}$ NMR (100 MHz, D6-DMSO, $\left.25{ }^{\circ} \mathrm{C}\right) \delta 169.8,169.3,168.4,168.1,164.9,151.4,142.6,136.2,136.0,128.9,128.9$, 128.7, 128.6, 128.5, 128.3, 108.7, 108.5, 67.1, 66.4, 49.5, 48.7, 48.5, 48.3, 47.2, 46.8, 12.3; HRMS (ESI) calculated for $\mathrm{C}_{18} \mathrm{H}_{20} \mathrm{~N}_{6} \mathrm{O}_{5}:\left(\mathrm{MH}^{+}\right): 401.1490$, found: 401.1500 .

\section{Benzyl $N$-(2-azidoethyl)- $N$-[4- $N$-(benzhydryloxycarbonyl)cytosine-1-acetyl]glycinate}

4-C. To a solution of 4- $N$-(benzhydryloxycarbonyl)cytosine-1-acetic acid 3-C (5.31 g, $14.0 \mathrm{mmol}, 1.2 \mathrm{eq})$ and $\mathrm{NMM}(3.26 \mathrm{~mL}, 25.6 \mathrm{mmol}, 2.2 \mathrm{eq})$ in DMF/acetonitrile (1/1, $33.3 \mathrm{~mL})$ at $0{ }^{\circ} \mathrm{C}$ was added pivaloyl chloride $(1.72 \mathrm{~mL}, 14.0 \mathrm{mmol}, 1.2 \mathrm{eq})$ drop-wise. The mixture was stirred for $20 \mathrm{~min}$ at $0{ }^{\circ} \mathrm{C}$ then benzyl 2-azidoethylglycinate $2(2.73 \mathrm{~g}$, $11.6 \mathrm{mmol}, 1.0 \mathrm{eq}$ ) was added and the reaction stirred $90 \mathrm{~min}$ at room temperature. The 
reaction was then diluted with $\mathrm{CH}_{2} \mathrm{Cl}_{2}$ and washed with $20 \%$ aq citric acid $(50 \mathrm{~mL})$ and saturated $\mathrm{NaHCO}_{3}(100 \mathrm{~mL})$. The organic phase was dried over $\mathrm{MgSO}_{4}$, concentrated and purified by flash chromatography (100\% EtOAc) to recover $4.01 \mathrm{~g}$ of 4-C as a white powder (50\% yield). $\quad R_{f}=0.80\left(10 \% \mathrm{MeOH}\right.$ in EtOAc); FT-IR (neat) $v_{\max } 3032.1$, 2944.7, 2103.6, 1744.5, 1668.6, 1628; ${ }^{1} \mathrm{H}$ NMR (400 MHz, D6-DMSO, $\left.25{ }^{\circ} \mathrm{C}\right)$ $\delta 11.01(\mathrm{~s}, 1 \mathrm{H}, \mathrm{CO}-\mathrm{NH}-\mathrm{C}), 7.92\left(\mathrm{~d}, J=7.5,0.7 \mathrm{H}, C_{6}\right.$, rotamer 1$), 7.85(\mathrm{~d}, J=7.5,0.3 \mathrm{H}$, $C_{6}$, rotamer 2), $7.39(\mathrm{~m}, 15 \mathrm{H}, \mathrm{Ar}), 6.96\left(\mathrm{~m}, 1 \mathrm{H}, C_{5}\right), 6.81\left(\mathrm{~s}, 1 \mathrm{H}, \mathrm{O}-\mathrm{CH}-\left(\mathrm{C}_{6} \mathrm{H}_{5}\right)_{2}\right), 5.23(\mathrm{~s}$, $0.7 \mathrm{H}, \mathrm{O}-\mathrm{CH}_{2}-\mathrm{C}_{6} \mathrm{H}_{5}$, rotamer 2), $5.14\left(\mathrm{~s}, 1.3 \mathrm{H}, \mathrm{O}-\mathrm{CH}_{2}-\mathrm{C}_{6} \mathrm{H}_{5}\right.$, rotamer 1), 4.89 (s, 1.3H, N$\mathrm{CH}_{2}-\mathrm{CO}$, rotamer 1), $4.69\left(\mathrm{~s}, 0.7 \mathrm{H}, \mathrm{N}-\mathrm{CH}_{2}-\mathrm{CO}\right.$, rotamer 2), 4.52 (s, $0.7 \mathrm{H}, \mathrm{N}-\mathrm{CH}_{2}-\mathrm{CO}$, rotamer 2), $4.19\left(\mathrm{~s}, 1.3 \mathrm{H}, \mathrm{N}-\mathrm{CH}_{2}-\mathrm{CO}\right.$, rotamer 1), $3.67\left(\mathrm{~m}, 2.6 \mathrm{H}, \mathrm{N}_{3}-\mathrm{CH}_{2}-\mathrm{CH}_{2}\right.$, rotamer 1), $3.51\left(\mathrm{t}, J=5.4,0.7 \mathrm{H}, \mathrm{N}_{3}-\mathrm{CH}_{2}-\mathrm{CH}_{2}\right.$, rotamer 2), 3.43 (t, $J=5.36,0.7 \mathrm{H}, \mathrm{CH}_{2}-\mathrm{CH}_{2}-\mathrm{N}$, rotamer 2); ${ }^{13} \mathrm{C}$ NMR (100 MHz, D6-DMSO, $\left.25{ }^{\circ} \mathrm{C}\right) \delta 169.8,169.3,168.3,168.0,163.5$, $155.4,152.8,151.5,140.8,136.2,136.0,129.0,128.9,128.6,128.5,128.3,126.9,94.2$, 77.9, 67.0, 66.4, 50.1, 49.5, 48.8, 48.6, 47.4, 46.8; HRMS (ESI) calculated for $\mathrm{C}_{31} \mathrm{H}_{29} \mathrm{~N}_{7} \mathrm{O}_{6}\left(\mathrm{MH}^{+}\right)$: 596.2174, found: 596.2157.

\section{Benzyl $N$-(2-azidoethyl)- $N$-[6- $N$-(benzhydryloxycarbonyl)adenine-9-acetyl]glycinate}

4-A. A solution of 6- $N$-(benzhydryloxycarbonyl)adenine-9-acetic acid 3-A (0.20 g, 0.50 mmol, $1.0 \mathrm{eq})$ in acetonitrile at $0{ }^{\circ} \mathrm{C}(1.3 \mathrm{~mL})$ was treated sequentially with NMM (111 $\mu \mathrm{L}, 0.99 \mathrm{mmol}, 2.0 \mathrm{eq})$ and pivaloyl chloride as a drop-wise addition $(67.2 \mu \mathrm{L}, 0.55$ mmol, 1.2 eq). The mixture was stirred for $20 \mathrm{~min}$ at $0{ }^{\circ} \mathrm{C}$ then benzyl 2azidoethylglycinate $2(0.12 \mathrm{~g}, 0.51 \mathrm{mmol}, 1.02 \mathrm{eq})$ was added and the reaction was continued for $90 \mathrm{~min}$ at room temperature. The reaction was then diluted with $\mathrm{CH}_{2} \mathrm{Cl}_{2}$ and washed with $20 \%$ aq citric acid followed by saturated $\mathrm{NaHCO}_{3}$. The organic phase was dried over $\mathrm{MgSO}_{4}$, concentrated and purified by flash chromatography $(10 \%$ $\mathrm{MeOH} / \mathrm{EtOAc})$ to obtain $0.522 \mathrm{~g}$ of $\mathbf{4 A}$ as white powder ( $80 \%$ yield). $R_{f}=0.65(10 \%$ $\mathrm{MeOH}$ in EtOAc); FT-IR (neat) $v_{\max } 3397.3,2927.1,2105.3,1751,1675,1612 \mathrm{~cm}^{-1}$; ${ }^{1} \mathrm{H}$ NMR (400 MHz, D6-DMSO, $\left.25{ }^{\circ} \mathrm{C}\right) \delta 8.61\left(1 \mathrm{~s}, 1 \mathrm{H}, C_{2}\right), 8.38\left(\mathrm{~s}, 0.7 \mathrm{H}, C_{8}\right.$, rotamer 1$)$, $8.33\left(\mathrm{~s}, 0.3 \mathrm{H}, C_{8}\right.$, rotamer 2), 7.56-7.30 (m, 15H, Ar), $6.83\left(\mathrm{~s}, 1 \mathrm{H}, \mathrm{O}-\mathrm{CH}-\left(\mathrm{C}_{6} \mathrm{H}_{5}\right)_{2}\right), 5.50$ $\left(\mathrm{s}, 1.3 \mathrm{H}, \mathrm{Ph}-\mathrm{CH}_{2}-\mathrm{CON}\right.$, rotamer 1), $5.28\left(\mathrm{~s}, 0.7 \mathrm{H}, \mathrm{Ph}-\mathrm{CH}_{2}-\mathrm{CON}\right.$, rotamer 2), $\underline{6}: 5.25$ (s, $0.6 \mathrm{H}, \mathrm{N}-\mathrm{CH}_{2}-\mathrm{CON}$, rotamer 2), $5.13\left(\mathrm{~s}, 1.4 \mathrm{H}, \mathrm{N}-\mathrm{CH}_{2}-\mathrm{CON}\right.$, rotamer 1$), 4.63(\mathrm{~s}, 0.7 \mathrm{H}$, 
$\mathrm{NCH}_{2} \mathrm{CO}_{2}$, rotamer 2), $4.21\left(\mathrm{~s}, 1.3 \mathrm{H}, \mathrm{NCH}_{2} \mathrm{CO}_{2}\right.$, rotamer 1), $3.74\left(\mathrm{~m}, 2.6 \mathrm{H}, \mathrm{N}_{3}-\mathrm{CH}_{2}-\mathrm{CH}_{2}-\right.$ $\mathrm{N}$, rotamer 1), $3.53\left(\mathrm{t}, J=4.8,0.6 \mathrm{H}, \mathrm{N}_{3}-\mathrm{CH}_{2}-\mathrm{CH}_{2}-\mathrm{N}\right.$, rotamer 2), $3.45(\mathrm{t}, J=4.8,0.6 \mathrm{H}$, N3-CH2-CH2-N, rotamer 2); ${ }^{13} \mathrm{C}$ NMR (100 MHz, D6-DMSO, $\left.25{ }^{\circ} \mathrm{C}\right) \delta 169.8,169.3$, $167.8,167.5,152.8,152.0,151.6,149.7,145.7,141.4,136.2,128.9,128.6,128.5,128.3$, $128.1,126.9,123.1,77.7,67.6,66.4,49.6,48.7,48.6,47.4,46.9,44.5$; HRMS (ESI) calculated for $\mathrm{C}_{32} \mathrm{H}_{29} \mathrm{~N}_{9} \mathrm{O}_{5}\left(\mathrm{MH}^{+}\right)$: 620.2286 , found: 620.2113 .

\section{Benzyl}

$N$-(2-azidoethyl)- $N$-[2- $N$-(benzhydryloxycarbonyl)-guanine-9-

acetyl]glycinate 4-G. To a solution of 2-N-(benzhydryloxycarbonyl)-guanine-9-acetatic acid 3-G (0.40 g, $0.96 \mathrm{mmol}, 1.1 \mathrm{eq})$ in DMF (2 mL), was added TOTU (0.32 g, 0.96 mmol, $1.1 \mathrm{eq})$ followed by $\mathrm{Et}_{3} \mathrm{~N}(0.2 \mathrm{~mL}, 1.50 \mathrm{mmol}, 1.7 \mathrm{eq})$. The mixture was stirred for $10 \mathrm{~min}$ and benzyl 2-azidoethylglycinate $2(0.2 \mathrm{~g}, 0.87 \mathrm{mmol}, 1.0 \mathrm{eq})$ was added. After 2 $\mathrm{h}$, the reaction was diluted with $\mathrm{CH}_{2} \mathrm{Cl}_{2}(50 \mathrm{~mL})$ and washed with brine $(100 \mathrm{~mL}), 20 \%$ aq citric acid $(50 \mathrm{~mL}), \mathrm{NaHCO}_{3}(100 \mathrm{~mL})$, dried over $\mathrm{MgSO}_{4}$ and concentrated. The crude product was purified by flash chromatography $(10 \% \mathrm{MeOH}$ in EtOAc) to recover $0.27 \mathrm{~g}$ of $4-\mathbf{G}$ as a white powder (49\% yield). $R_{f}=0.30(10 \% \mathrm{MeOH}$ in EtOAc); FT-IR $\left(\right.$ KBr pellet) $v_{\max } 3245.4,2925.1,2104.5,1685.7,1609.1 \mathrm{~cm}^{-1} ;{ }^{1} \mathrm{H}$ NMR (400 MHz, D6-DMSO, $\left.25^{\circ} \mathrm{C}\right) \delta 7.86\left(\mathrm{~s}, 1 \mathrm{H}, \mathrm{C}_{8}\right), 7.48-7.30(\mathrm{~m}, 15 \mathrm{H}, \mathrm{Ar}), 6.88(\mathrm{~s}, 1 \mathrm{H}, \mathrm{O}-\mathrm{CH}-\mathrm{Ph})$, $5.26\left(\mathrm{~s}, 0.8 \mathrm{H}, \mathrm{N}-\mathrm{CH}_{2}-\mathrm{CO}\right.$, rotamer 1), $5.21\left(\mathrm{~s}, 1.2 \mathrm{H}, \mathrm{N}-\mathrm{CH}_{2}-\mathrm{CO}\right.$, rotamer 2), 5.14 (s, $1.4 \mathrm{H}, \mathrm{O}-\mathrm{CH}_{2}-\mathrm{Ar}_{\mathrm{Bn}}$, rotamer 1), $5.03\left(\mathrm{~s}, 0.6 \mathrm{H}, \mathrm{O}-\mathrm{CH}_{2}-\mathrm{Bn}\right.$ rotamer 2$), 3.72\left(\mathrm{~s}, 2 \mathrm{H}, \mathrm{N}-\mathrm{CH}_{2^{-}}\right.$ $\mathrm{CO}), 3.71\left(\mathrm{~m}, 2 \mathrm{H}, \mathrm{N}_{3}-\mathrm{CH}_{2}-\mathrm{CH}_{2}\right.$, rotamer 1), $3.71\left(\mathrm{~m}, 2 \mathrm{H}, \mathrm{CH}_{2}-\mathrm{CH}_{2}-\mathrm{N}\right.$, rotamer 1), 3.52 $\left(\mathrm{t}, J=5.4,0.5 \mathrm{H}, \mathrm{N}_{3}-\mathrm{CH}_{2}-\mathrm{CH}_{2}\right.$, rotamer 2), $3.44\left(\mathrm{t}, J=5.4,0.5 \mathrm{H}, \mathrm{CH}_{2}-\mathrm{CH}_{2}-\mathrm{N}\right.$, rotamer 2); ${ }^{13} \mathrm{C}$ NMR (100 MHz, D6-DMSO, $\left.25{ }^{\circ} \mathrm{C}\right) \delta 170.8,169.9,169.3,167.4,167.5,155.5$, $154.1,147.4,141.0,140.5,136.2,136.0,129.0,128.9,128.8,128.6,128.4,128.3,78.5$, 67.2, 66.4, 53.0, 49.0, 48.7, 47.3, 46.9, 44.5, 44.6; HRMS (ESI) calculated for $\mathrm{C}_{32} \mathrm{H}_{29} \mathrm{~N}_{9} \mathrm{O}_{6}\left(\mathrm{MH}^{+}\right)$: 636.2235, found: 636.2055.

$\mathrm{N}$-(2-Azidoethyl)- $\mathrm{N}$-(thymine-1-acetyl)glycine 5-T. To a solution of benzyl ester 4-T $(14.8 \mathrm{~g}, 37.1 \mathrm{mmol}, 1.0 \mathrm{eq})$ in 1,4-dioxane $(35 \mathrm{~mL})$ at room temperature was added $\mathrm{NaOH}$ as a $2 \mathrm{M}$ solution $(74.2 \mathrm{~mL}, 148.5 \mathrm{mmol}, 4.0 \mathrm{eq})$. After $15 \mathrm{~min}$, TLC analysis indicated that the reaction was complete and $20 \mathrm{~mL}$ of dioxane were evaporated. The 
solution was acidified until $\mathrm{pH} 3$ with $20 \%$ aq citric acid at which stage the desired product precipitated. Filtration followed by drying in vaccuo afforded $8.88 \mathrm{~g}$ as a pure white solid (77\% yield). $\quad R_{f}=0.08\left(30 \% \mathrm{MeOH}\right.$ in EtOAc); FT-IR (neat) $v_{\max }$ 3448.1,3171.7, 3041.3, 2105.9, 1696.4, $1655.2 \mathrm{~cm}^{-1}$; ${ }^{1} \mathrm{H}$ NMR (400 MHz, D6-DMSO, $\left.25^{\circ} \mathrm{C}\right) \delta 7.30\left(\mathrm{bs}, 0.5 \mathrm{H}, C_{6}\right.$, rotamer 1$), 7.26\left(\mathrm{bs}, 0.5 \mathrm{H}, C_{6}\right.$, rotamer 2$), 4.71(\mathrm{~s}, 1.1 \mathrm{H}, \mathrm{N}-$ $\mathrm{CH}_{2}-\mathrm{CO}_{2}$, rotamer 1), $4.49\left(\mathrm{~s}, 0.9 \mathrm{H}, \mathrm{N}-\mathrm{CH}_{2}-\mathrm{CO}_{2}\right.$, rotamer 2), $3.88\left(\mathrm{~s}, 1.1 \mathrm{H}, \mathrm{N}-\mathrm{CH}_{2}-\mathrm{CON}\right.$, rotamer 2), $3.86\left(\mathrm{~s}, 0.9 \mathrm{H}, \mathrm{N}-\mathrm{CH}_{2}-\mathrm{CON}\right.$, rotamer 1), 3.55 (t, $J=4.8,1 \mathrm{H}, \mathrm{N}_{3}-\mathrm{CH}_{2}-\mathrm{CH}_{2}$, rotamer 1), 3.47 (t, $J=4.8,1 \mathrm{H}, \mathrm{N}_{3}-\mathrm{CH} 2-\mathrm{CH}_{2}$, rotamer 1), 3.41 (t, $J=5.4,1 \mathrm{H}, \mathrm{N}_{3}-\mathrm{CH}_{2^{-}}$ $\mathrm{CH}_{2}$, rotamer 2), 3.36 (t, $J=5.4,1 \mathrm{H}, \mathrm{N}_{3}-\mathrm{CH}_{2}-\mathrm{CH}_{2}-\mathrm{N}$, rotamer 2), 1.75 (s, $3 \mathrm{H}, \mathrm{C}-\mathrm{CH}_{3}$ ); ${ }^{13} \mathrm{C}$ NMR (100 MHz, D6-DMSO, $\left.25{ }^{\circ} \mathrm{C}\right) \delta 171.3,170.8,168.4,167.8,164.9,151.4$, 142.7, 142.6, 108.6, 50.2, 49.4, 48.7, 48.3, 47.0, 46.8, 12.3; HRMS (ESI) calculated for $\mathrm{C}_{11} \mathrm{H}_{14} \mathrm{~N}_{6} \mathrm{O}_{5}\left(\mathrm{MH}^{+}\right): 311.1020$, found: 311.1075 .

$\mathrm{N}$-(2-Azidoethyl)- $\mathrm{N}$-[4- $\mathrm{N}$-(benzhydryloxycarbonyl)cytosine-1-acetyl]glycine 5-C. A solution of benzyl ester 4-C (21.0 g, $35.2 \mathrm{mmol}, 1.0 \mathrm{eq})$ in 1,4-dioxane $(35 \mathrm{~mL})$ at room temperature was treated with $\mathrm{NaOH} 2 \mathrm{M}(70.4 \mathrm{~mL}, 140.8 \mathrm{mmol}, 4.0 \mathrm{eq})$. After $15 \mathrm{~min}$, TLC analysis indicated that the reaction had proceeded to completion and the solution was acidified until $\mathrm{pH} 3$ with $20 \%$ aq citric acid which resulted in the precipitation of the desired acid. Filtration followed by drying in vaccuo afforded $16.8 \mathrm{~g}$ of acid 5-C as pure white powder (95\% yield). $R_{f}=0.19$ (30\% MeOH in EtOAc); FT-IR (neat) $v_{\max } 3227.5$, 3029.7, 2106.2, 1740, 1633.8, 1572, $1507 \mathrm{~cm}^{-1}$; ${ }^{1} \mathrm{H}$ NMR (400 MHz, D6-DMSO, $2{ }^{\circ} \mathrm{C}$ ) $\delta 7.94\left(\mathrm{~d}, 0.6 \mathrm{H}, J=7.5, C_{6}\right.$, rotamer 1), $7.90\left(\mathrm{~d}, 0.4 \mathrm{H}, J=7.5, C_{6}\right.$, rotamer 2), $7.47(\mathrm{~d}, J$ $=7.5,4 \mathrm{H}, \mathrm{Ph}$ ), 7.38 (t, $J=7.5,4 \mathrm{H}, \mathrm{Ph}), 7.32$ (t, $2 \mathrm{H}, J=7.5, \mathrm{Ph}), 6.96$ (d, $0.6 \mathrm{H}, J=7.5$, $C_{5}$, rotamer 1), $6.95\left(\mathrm{~d}, 0.4 \mathrm{H}, J=7.5, C_{5}\right.$, rotamer 2), $6.81\left(\mathrm{~s}, 1 \mathrm{H}, \mathrm{O}-\mathrm{CH}-\left(\mathrm{C}_{6} \mathrm{H}_{5}\right)_{2}\right), 4.87$ (s, $1.2 \mathrm{H}, \mathrm{N}-\mathrm{CH}_{2}-\mathrm{CO}$, rotamer 1), $4.66\left(\mathrm{~s}, 0.8 \mathrm{H}, \mathrm{N}-\mathrm{CH}_{2}-\mathrm{CO}\right.$, rotamer 2$), 4.03(\mathrm{~s}, 1.2 \mathrm{H}, \mathrm{N}-$ $\mathrm{CH}_{2}-\mathrm{CO}$, rotamer 2), 4.32 (s, $0.8 \mathrm{H}, \mathrm{N}-\mathrm{CH}_{2}-\mathrm{CO}$, rotamer 1$), 3.66\left(\mathrm{t}, J=4.8,1.2 \mathrm{H}, \mathrm{N}_{3-}\right.$ $\mathrm{CH}_{2}-\mathrm{CH}_{2}$ rotamer 1), 3.61 (t, $J=4.8,1.2 \mathrm{H}, \mathrm{CH}_{2}-\mathrm{CH}_{2} \mathrm{-N}$, rotamer 1), 3.50 (t, $J=5.4$, $0.8 \mathrm{H}, \mathrm{N}_{3}-\mathrm{CH}_{2}-\mathrm{CH}_{2}$, rotamer 2), $3.45\left(\mathrm{t}, J=5.4,0.8 \mathrm{H}, \mathrm{CH}_{2}-\mathrm{CH}_{2}-\mathrm{N}\right.$, rotamer 2$) ;{ }^{13} \mathrm{C} \mathrm{NMR}$ $\left(100 \mathrm{MHz}, \mathrm{D} 6-\mathrm{DMSO}, 25^{\circ} \mathrm{C}\right) \delta 171.2,170.8,168.2,167.7,163.5,155.4,152.8,151.4$, 143.0, 140.8, 129.0, 126.9, 128.5, 94.3, 77.9, 50.1, 50.1, 49.5, 49.7, 47.2, 46.8; HRMS (ESI) calculated for $\mathrm{C}_{24} \mathrm{H}_{23} \mathrm{~N}_{7} \mathrm{O}_{6}\left(\mathrm{MH}^{+}\right)$: 506.1704, found: 506.1606 . 
$\mathrm{N}$-(2-Azidoethyl)- $\mathrm{N}$-[6- $N$-(benzhydryloxycarbonyl)adenine-9-acetyl]glycine 5-A. To a solution of benzyl ester 4-A $(0.25 \mathrm{~g}, 0.39 \mathrm{mmol}, 1.0 \mathrm{eq})$ in dioxane $(1 \mathrm{~mL})$ at room temperature was added $\mathrm{NaOH} 2 \mathrm{M}(0.79 \mathrm{~mL}, 1.58 \mathrm{mmol}, 4.0$ eq). After $15 \mathrm{~min}$, all starting material had been consumed as jugged by TLC and the desired compound was precipitated by acidification to $\mathrm{pH} 3$ with $20 \%$ aq citric acid. Filtration of the white precipitate and drying in vaccuo afforded $0.16 \mathrm{~g}$ of acid 5-A as a pure white solid (76\% yield). $R_{f}=0.14\left(30 \% \mathrm{MeOH}\right.$ in EtOAc); FT-IR (neat) $v_{\max }$ 3031.8, 2925.9, 2103.4, 1763.6, 1671.1, $1616 \mathrm{~cm}^{-1}$; ${ }^{1} \mathrm{H}$ NMR (400 MHz, D6-DMSO, $\left.25^{\circ} \mathrm{C}\right) \delta 8.61\left(\mathrm{~s}, 0.6 \mathrm{H}, C_{2}\right.$, rotamer 1$), 8.60\left(\mathrm{~s}, 0.4 \mathrm{H}, C_{2}\right.$, rotamer 2$), 8.39\left(\mathrm{~s}, 0.6 \mathrm{H}, C_{8}\right.$, rotamer 1$), 8.36\left(\mathrm{~s}, 0.4 \mathrm{H}, C_{8}\right.$, rotamer 2), $7.54(\mathrm{~d}, J=7.5,4 \mathrm{H}, \mathrm{Ph}) ; 7.39$ (t, $J=7.5,4 \mathrm{H}, \mathrm{Ph}), 7.30(\mathrm{t}, J=7.5,2 \mathrm{H}, \mathrm{Ph})$, $6.83\left(\mathrm{~s}, 1 \mathrm{H}, \mathrm{O}-\mathrm{CH}-\left(\mathrm{C}_{6} \mathrm{H}_{5}\right)_{2}\right), 5.43\left(\mathrm{~s}, 1.2 \mathrm{H}, \mathrm{N}-\mathrm{CH}_{2}-\mathrm{CO}\right.$, rotamer 1), $5.20\left(\mathrm{~s}, 0.8 \mathrm{H}, \mathrm{N}-\mathrm{CH}_{2^{-}}\right.$ $\mathrm{CO}$, rotamer 2), ㅁ: $4.39\left(\mathrm{~s}, 0.8 \mathrm{H}, \mathrm{N}-\mathrm{CH}_{2}-\mathrm{CO}\right.$, rotamer 1), $4.05\left(\mathrm{~s}, 1.2 \mathrm{H}, \mathrm{N}-\mathrm{CH}_{2}-\mathrm{CO}\right.$, rotamer 2), $3.72\left(\mathrm{~m}, 2.4 \mathrm{H}, \mathrm{N}_{3}-\mathrm{CH}_{2}-\mathrm{CH}_{2}-\mathrm{N}\right.$, rotamer 1), 3.50 (t, $\mathrm{J}=5.4,0.8 \mathrm{H}, \mathrm{N}_{3}-\mathrm{CH}_{2}-$ $\mathrm{CH}_{2}$, rotamer 2), $3.46\left(\mathrm{t}, J=5.4,0.8 \mathrm{H}, \mathrm{CH}_{2}-\mathrm{CH}_{2}-\mathrm{N}\right.$, rotamer 2$) ;{ }^{13} \mathrm{C} \mathrm{NMR}(100 \mathrm{MHz}$, D6-DMSO, $\left.25^{\circ} \mathrm{C}\right) \delta 171.3,170.8,167.8,167.2,152.8,152.0,151.6,149.7,145.7,141.3$, 128.9, 128.1, 126.9, 123.1, 77.7, 49.5, 48.7, 48.3, 47.2, 46.9, 44.5; HRMS (ESI) calculated for $\mathrm{C}_{25} \mathrm{H}_{23} \mathrm{~N}_{9} \mathrm{O}_{5}$ : $\left(\mathrm{MH}^{+}\right)$: 530.1817, found: 530.1787 .

$N$-(2-Azidoethyl)- $N$-[2- $N$-(benzhydryloxycarbonyl)-guanine-9-acetyl]glycine 5-G. To a solution of benzyl ester 4-G $(0.18 \mathrm{~g}, 283 \mu \mathrm{mol}, 1.0 \mathrm{eq})$ in 1,4-dioxane $(725 \mu \mathrm{L})$ was added $\mathrm{NaOH} 2 \mathrm{M}(566 \mu \mathrm{L}, 1.13 \mathrm{mmol}, 4.0 \mathrm{eq})$. After $20 \mathrm{~min}$, the solution was acidified until pH 3 with $20 \%$ aq citric acid, were the compound precipited. Filtration of the white precipitate and drying in vacuo afforded $123 \mathrm{mg}$ of desire acid 5-G as a white solid (80\% yield). $\quad R_{f}=0.11\left(30 \% \mathrm{MeOH}\right.$ in EtOAc); FT-IR (neat) $v_{\max } 3407,3237.4,2946.1$, 2103.4, 1678.3, 1611.9, $1572 \mathrm{~cm}^{-1} ;{ }^{1} \mathrm{H}$ NMR (400 MHz, D6-DMSO, $\left.25^{\circ} \mathrm{C}\right) \delta 7.87(\mathrm{~s}$, $0.6 \mathrm{H}, C_{8}$, rotamer 1), $8.83\left(\mathrm{~s}, 0.4 \mathrm{H}, C_{8}\right.$, rotamer 2$), 7.48(\mathrm{~d}, J=7.5,4 \mathrm{H}, \mathrm{Ph}), 7.40(\mathrm{t}, J=$ $7.5,4 \mathrm{H}, \mathrm{Ph}), 7.32(\mathrm{t}, J=7.5,2 \mathrm{H}, \mathrm{Ph}), 6.88\left(\mathrm{~s}, 1 \mathrm{H}, \mathrm{O}-\mathrm{CH}-\left(\mathrm{C}_{6} \mathrm{H}_{5}\right)_{2}\right), 5.18\left(\mathrm{~s}, 1.2 \mathrm{H}, \mathrm{N}-\mathrm{CH}_{2}-\right.$ $\mathrm{CON}$, rotamer 1), $4.98\left(\mathrm{~s}, 0.8 \mathrm{H}, \mathrm{N}-\mathrm{CH}_{2}-\mathrm{CON}\right.$, rotamer 2), $4.40\left(\mathrm{~s}, 0.7 \mathrm{H}, \mathrm{N}-\mathrm{CH}_{2}-\mathrm{CO}_{2}\right.$, rotamer 2), $4.05\left(\mathrm{~s}, 1.3 \mathrm{H}, \mathrm{N}-\mathrm{CH}_{2}-\mathrm{CO}_{2}\right.$, rotamer 1$), 3.71\left(\mathrm{t}, \mathrm{J}=4.8,1.2 \mathrm{H}, \mathrm{N}_{3}-\mathrm{CH}_{2}-\mathrm{CH}_{2}\right.$, rotamer 1), $3.66\left(\mathrm{t}, J=4.8,1.2 \mathrm{H}, \mathrm{N}_{3}-\mathrm{CH}_{2}-\mathrm{CH}_{2}\right.$, rotamer 1$), 3.48\left(\mathrm{t}, J=5.4,0.8 \mathrm{H}, \mathrm{N}_{3}-\right.$ 
$\mathrm{CH}_{2}-\mathrm{CH}_{2}$, rotamer 2), $3.45\left(\mathrm{t}, \mathrm{J}=5.4,0.8 \mathrm{H}, \mathrm{CH}_{2}-\mathrm{CH}_{2}-\mathrm{N}\right.$, rotamer 2$) ;{ }^{13} \mathrm{C}$ NMR (100 $\left.\mathrm{MHz}, \mathrm{D} 6-\mathrm{DMSO}, 25^{\circ} \mathrm{C}\right) \delta 171.2,170.7,169.5,167.7,167.2,155.5,154.2,146.9,149.7$, $147.4,147.6,141.1,140.9,140.5,140.4,129.0,128.4,126.9,78.5,49.7,49.4,48.7,48.3$, 47.1, 46.9, 44.8, 44.4; HRMS (ESI) calculated for $\mathrm{C}_{25} \mathrm{H}_{23} \mathrm{~N}_{9} \mathrm{O}_{6}:\left(\mathrm{MH}^{+}\right)$: 546.1766, found: 546.1713 .

\section{General procedure for oligomerization of azidoPNA.}

Azide reduction. The resin was treated with a $1 \mathrm{M} \mathrm{PMe}_{3}: \mathrm{H}_{2} \mathrm{O}(9: 1 ; 1 \mathrm{~mL} / \mathrm{g}$ of resin) for 5 min then washed with THF ( $3 \times 1 \mathrm{~mL} / \mathrm{g}$ of resin) and treated immediately with the pre-activated solution of acid $\mathbf{5}$.

Activation-coupling. A solution of acid 5 (3.0 eq) in DMF (0.1 M) was treated with DIC (2.5 eq) and HOBt (3.0 eq) and agitated for $1 \mathrm{~h}$ prior to reaction. The activated acid solution was added to the freshly reduced or otherwise deprotected resin and the reaction was allowed to proceed for $1 \mathrm{~h}$.

Cleavage. A sample of resin was treated with TFA: $m$ cresol (9:1) for $1 \mathrm{~h}$ then precipitated in $\mathrm{Et}_{2} \mathrm{O}$ and collected by centrifugation.

Synthesis of 6-mer PNA 14. Rink Amide resin loaded with Boc-protected lysine 13 was prepared using standard Fmoc chemistry. The PNA oligomerization was performed on $35 \mu \mathrm{mol}$ according to the general procedure described above with double coupling at each step and capping with $\mathrm{Ac}_{2} \mathrm{O} /$ pyridine (4.0 eq, $0.1 \mathrm{M}$ in DMF for $5 \mathrm{~min}$ ). An analytical sample was removed and cleaved at each step and analyzed by LC-MS. HRMS (ESI) calculated for: K-T $\left(\mathrm{MH}^{+}\right)$: 438.2130, found 438.2650; K-TC $\left(\mathrm{MH}^{+}\right)$: 689.3148, found 689.3639; K-TCA $\left(\mathrm{MH}^{+}\right)$: 964.4278, found 964.4671; K-TCAG $\left(\mathrm{MH}^{+}\right)$: 1255.5359, found 1255.5809; K-TCAGC $\left(\mathrm{MH}^{+}\right)$: 1506.6377, found 1506.6977; K-TCAGCT $\left(\mathrm{M} 2 \mathrm{H}^{+}\right)$: 887.3696, found $\left(\mathrm{M}-\mathrm{C} 13-\mathrm{H}^{++}\right)$887.4299. 

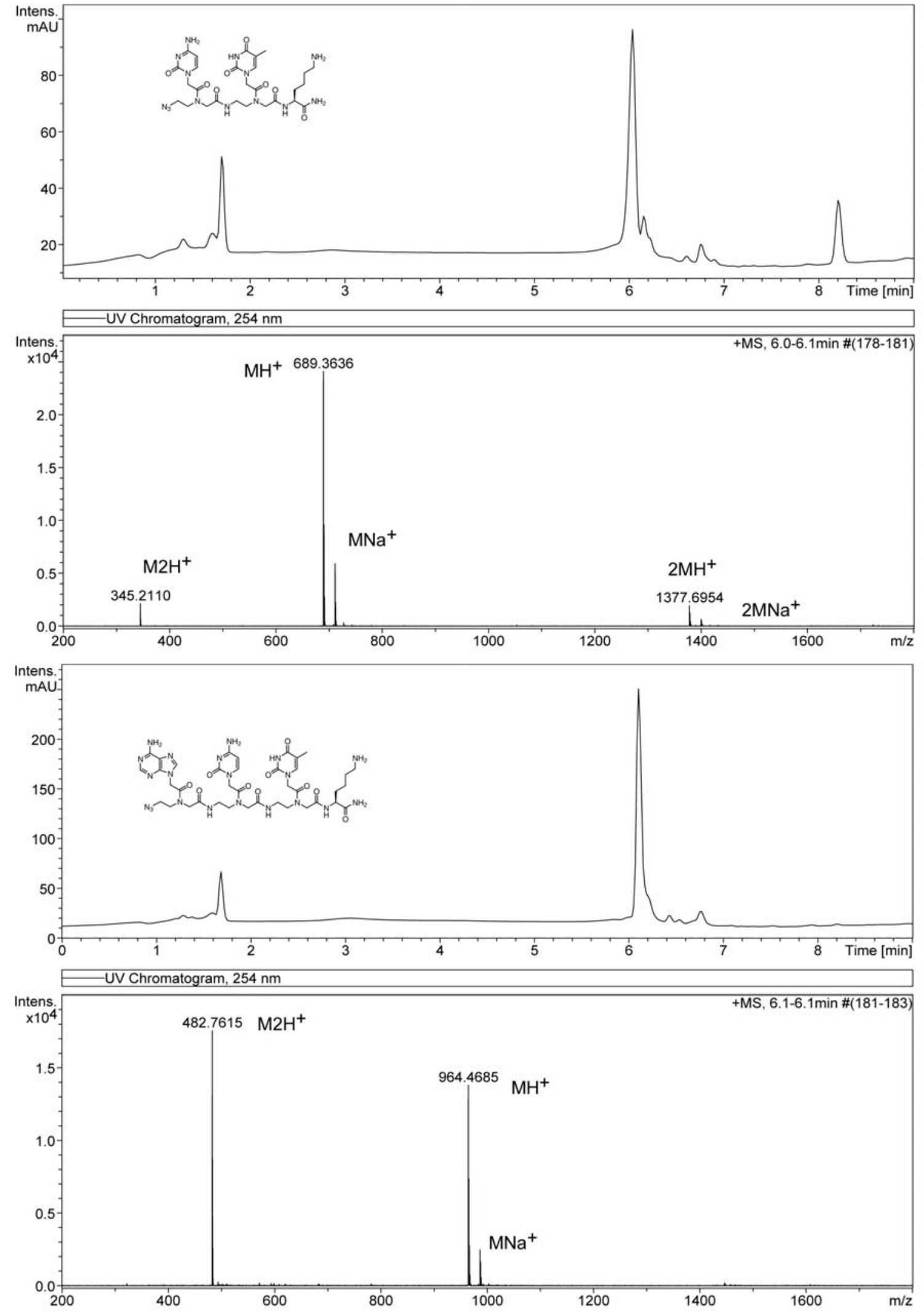

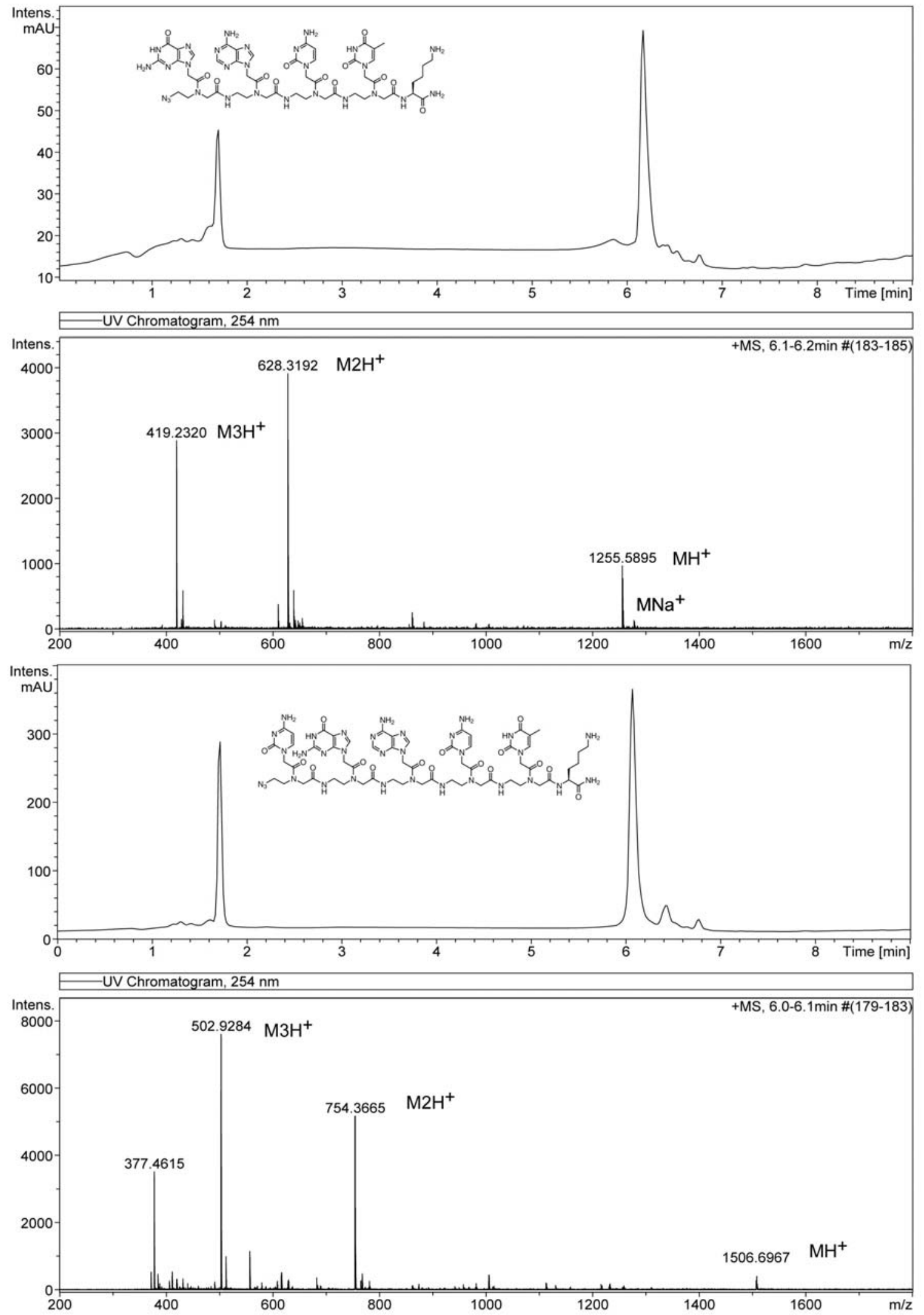

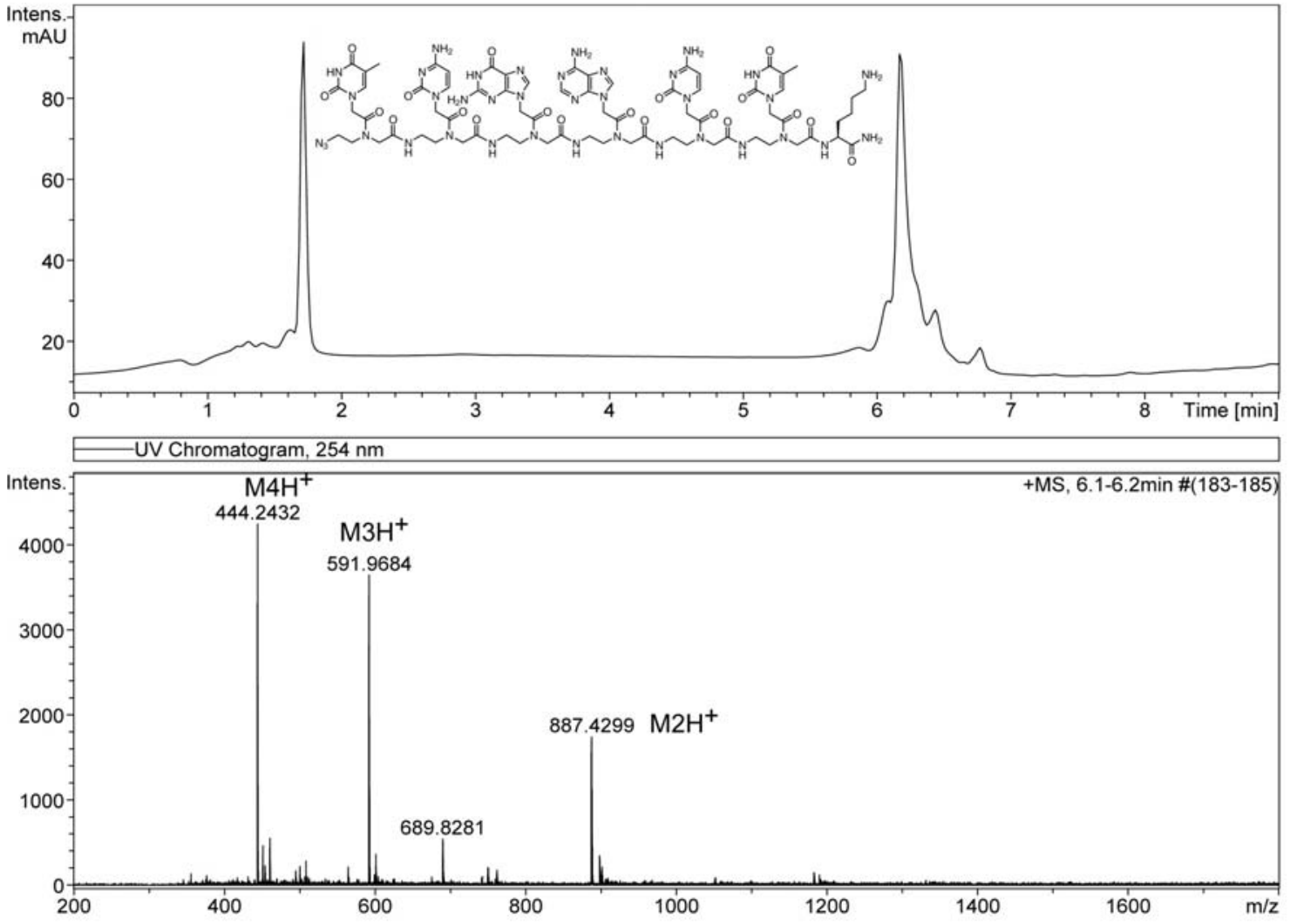